\title{
Laser-induced desorption of Na-dimers
}

\author{
J. Viereck ${ }^{1}$, M. Stuke ${ }^{2}$, F. Träger ${ }^{1}$ \\ ${ }^{1}$ Fachbereich Physik, Universität Kassel, Heinrich-Plett-Str. 40, D-34132 Kassel, Germany \\ (Fax: + 49-561-804-4518, E-mail: Traeger@physik.uni-kassel.de) \\ ${ }^{2}$ Max-Planck-Institut für biophysikalische Chemie, P.O.Box 2841, D-37018 Göttingen, Germany
}

Received: 23 July 1996 / Accepted: 26 August 1996

\begin{abstract}
We find that Na-dimers are desorbed in a thermal process if rough $\mathrm{Na}$ surfaces are irradiated with pulsed laser light of $\lambda=532 \mathrm{~nm}$. In contrast, for light of $\lambda=355 \mathrm{~nm}, \mathrm{Na}_{2}$ can be detached in a non-thermal reaction at low laser fluences. This is concluded from the kinetic energy distributions of the dimers determined by time-of-flight measurements using a second laser at $\lambda=248 \mathrm{~nm}$ for photoionization. The transition from nonthermal to thermal desorption at large fluences of the laser light can also be identified.
\end{abstract}

PACS: 61.80.Ba; 68.55.Jk; 79.20.Ds; 36.40

It is well known that atoms, dimers, trimers, even clusters with as many as 15 or 20 atoms can be liberated from solid surfaces under bombardment with energetic ions [1]. Such processes are initiated by the transfer of momentum carried by the incident ions. In addition to detailed investigations on ion sputtering, several studies in the past showed that desorption of aggregates with two or more atoms can be induced by laser light as well (see e.g. [2-12]). Beyond that, the dynamics of the desorption process, i.e. breaking of the surface chemical bond, has been studied using femtosecond laser pulses [6-8]. Only few studies, however, dealt with laser-induced desorption of aggregates with two or more atoms out of metal or semiconductor surfaces $[2,4,5,12]$. For example, Brewer et al. [2] studied nanosecond and femtosecond UV laser ablation of $\mathrm{CdTe}(100)$ surfaces. They used angularly resolved timeof-flight measurements to probe the velocity and angular distributions of desorbing $\mathrm{Cd}$ atoms as well as $\mathrm{Te}_{2}$ molecules and found that the time-of-flight distributions of both ejected products could be described by Maxwellians. Götz et al. [5] investigated the mechanisms of laserinduced desorption of $\mathrm{Na}$-atoms and dimers from rough $\mathrm{Na}$ surfaces. The results showed, that for a laser wavelength of $\lambda=355 \mathrm{~nm}$ and fluences above $40 \mathrm{~mJ} / \mathrm{cm}^{2}$ desorption of Na-dimers takes place. With increasing laser fluence the fraction of detached Na-dimers as compared to Na-atoms increases. The experimentally observed energy distributions suggested that the dimers were directly desorbed from the surface in a thermal process and not formed by gas phase collisions of desorbed atoms [5]. As a continuation of this work the present paper reports studies of Na-dimer desorption at low laser fluences. The objective was, first, to clarify in these and in future experiments under which conditions clusters $X_{n}$ (with $n \geqslant 2$ ) can be desorbed with laser light in a non-thermal process and, secondly, to elucidate the underlying mechanism.

In contrast to ion sputtering transfer of momentum can be ruled out as a possible source for desorption, if surfaces are irradiated with laser light. Instead, electronic excitation has to be accomplished as the first step. It is usually followed by rapid relaxation and generation of heat causing thermal bond breaking. Under certain conditions, however, the electron-phonon coupling can be reduced in strength making possible non-thermal desorption (see e.g. [13]) of atomic species [14-17] as a direct result of repulsive electronic excitation. Further, it turns out that desorption along these lines takes place preferentially from defects of the surface, i.e. from sites with particularly low coordination numbers $[13,18]$. Therefore, in order to study desorption of clusters, it seems desirable to use surfaces with the largest possible number of such sites. For this purpose surfaces with large roughness have been prepared here by the deposition of metal atoms on dielectric substrates held at low temperature. Like in earlier work on atom desorption [19], sodium absorbed on quartz served as a model system. Initially, the deposited atoms form small particles with the substrate defects acting as nucleation centers [20]. If the deposition is continued, the clusters finally grow together generating a film. Formation of small particles on the surface of dielectric substrates ensures optimum conditions for the experiments described here, i.e. the desorption signal is readily detectable and the roughness of the cluster surface together with the desorption rate is reproducible.

The experimental arrangement has been described in detail elsewhere [5] and basically consists of an ultrahigh-vacuum system with the sample, two lasers for stimulating desorption and photo-ionizing the desorption products, and a time-of-flight mass spectrometer. A thermal 

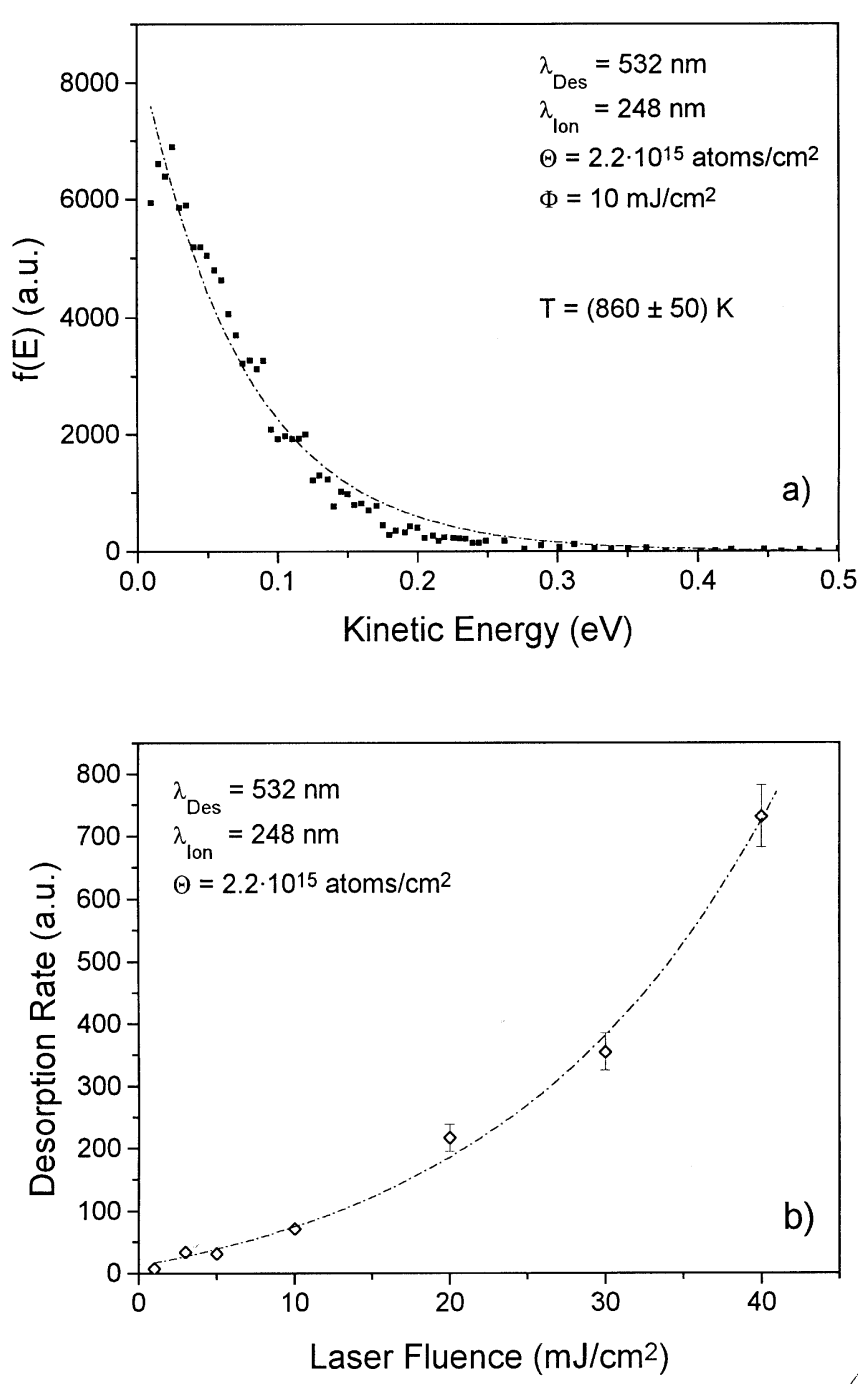

Fig. 1. a) Kinetic energy distribution of Na-dimers desorbed with laser light of $\lambda=532 \mathrm{~nm}$. The total coverage of sodium was held constant at $2.2 \cdot 10^{15}$ atoms $/ \mathrm{cm}^{2}$ which corresponds to an average particle size of $12.6 \mathrm{~nm}$. The fluence of the Nd:YAG laser was set to a value of $10 \mathrm{~mJ} / \mathrm{cm}^{2}$.

b) Integral desorption rate of Na-dimers desorbed with laser light of $\lambda=532 \mathrm{~nm}$ as a function of laser fluence

atomic beam of $\mathrm{Na}$ atoms with well-defined constant flux is directed onto the quartz substrate in order to deposit a predetermined coverage of atoms onto the surface held at $80 \mathrm{~K}$. The experiments reported here are carried out at low coverage and, in addition, at low laser fluence to keep the desorption rate small, avoid gas phase collisions of the desorbed species and thus measure their genuine kinetic energy distribution. After preparation the sample is irradiated with the light of a Nd:YAG laser at $\lambda=355$ or $532 \mathrm{~nm}$ in order to stimulate desorption. At a distance of $21 \mathrm{~mm}$ in front of the substrate the species detached from the cluster surface are ionized with the light of an excimer laser operating at $\lambda=248 \mathrm{~nm}$. The corresponding photon energy lies only little above the ionization threshold of $\mathrm{Na}_{2}$. Therefore, if compared to ealier work with $\lambda=193 \mathrm{~nm}$ where strong fragmentation of the Na dimers was observed [5], "soft" ionization of the desorbed dimers
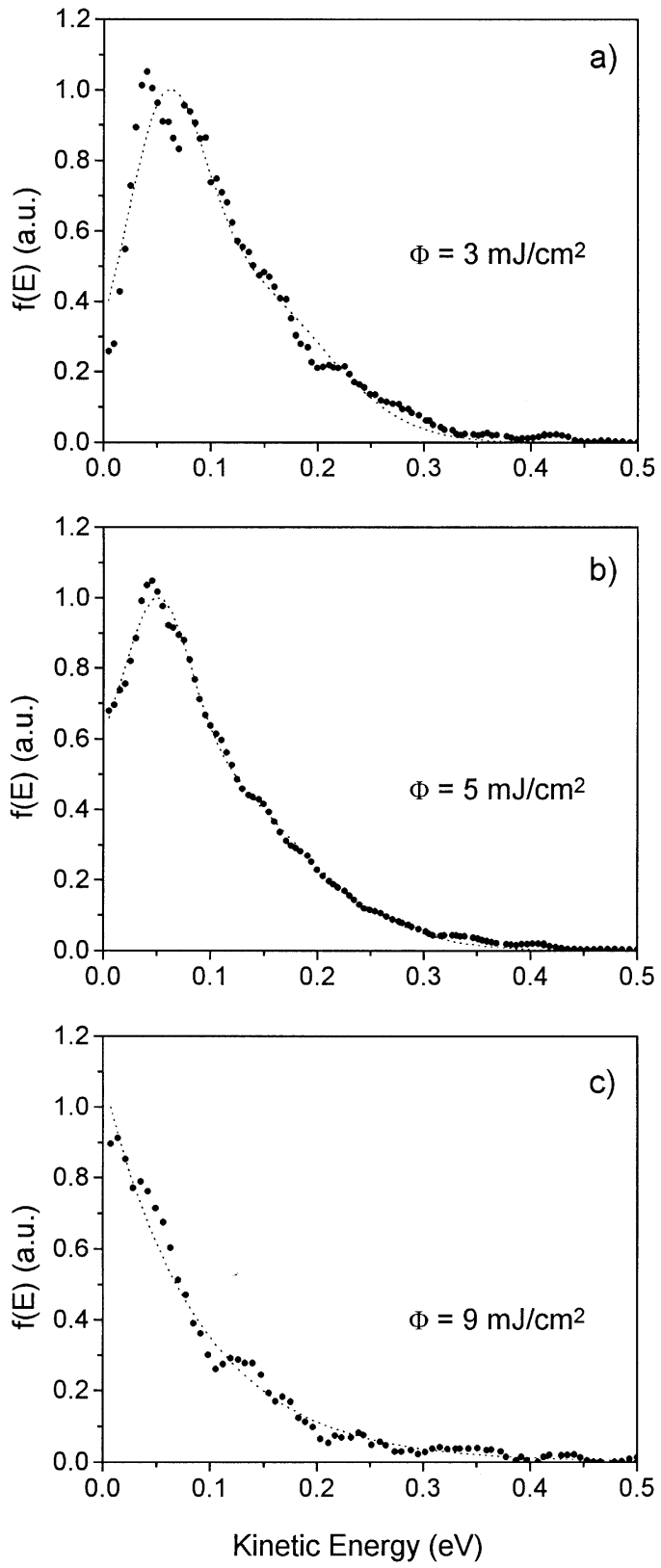

Fig. 2. Kinetic energy distributions normalized to the maximum desorption rate of Na-dimers desorbed at $\lambda=355 \mathrm{~nm}$ and a total coverage of $7.2 \cdot 10^{14}$ atoms $/ \mathrm{cm}^{2}\left(\mathrm{R}_{\mathrm{ave}}=7 \mathrm{~nm}\right)$ with fluences of a) $3 \mathrm{~mJ} / \mathrm{cm}^{2}$, b) $5 \mathrm{~mJ} / \mathrm{cm}^{2}$ and c) $9 \mathrm{~mJ} / \mathrm{cm}^{2}$ for an ionization wavelength of $\lambda=248 \mathrm{~nm}$.

is accomplished and there is only very little dissociation into monomers. The generated ions pass the time-of-flight mass spectrometer and are finally detected with a secondary electron multiplier. The ion signal is processed with a boxcar integrator and stored in a computer. By varying the delay time between the two laser pulses used for desorption and ionization the time-of-flight distributions of the desorbed Na-dimers are determined and then converted into flux distributions as a function of translational energy [21]. It should be mentioned that $\mathrm{Na}$ adsorbed on quartz is an ideal candidate for the measurements described here. This is not only because the 


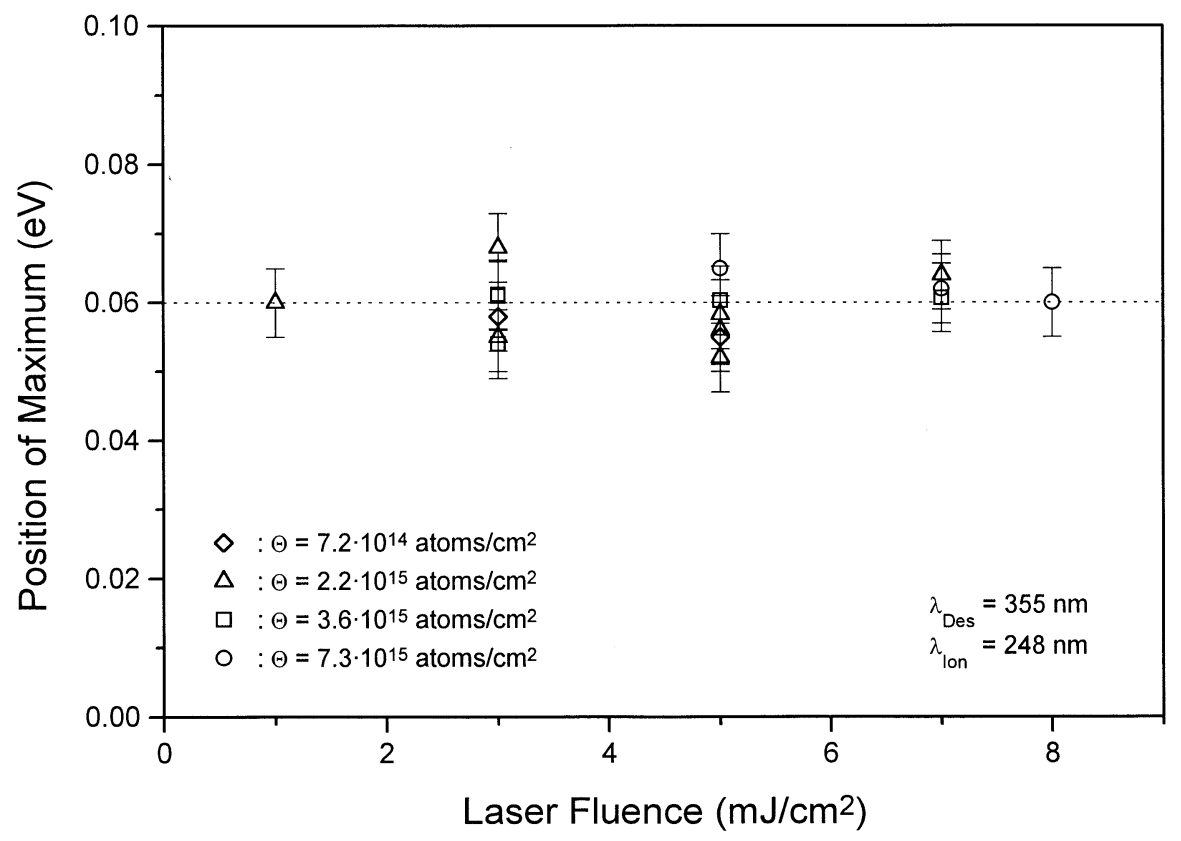

Fig. 3. Positions of the maxima of the kinetic energy distributions of Na-dimers desorbed with laser light of $\lambda=355 \mathrm{~nm}$. The laser fluence was varied between 1 and $8 \mathrm{~mJ} / \mathrm{cm}^{2}$ and the average particle size ranged from $7 \mathrm{~nm}$ to $15 \mathrm{~nm}$. Within experimental error the position of the maxima is identical and independent of laser fluence and mean particle size.

dimers can be ionized with a single laser photon but also because the coverage, after completion of a measurement, can be evaporated completely at a temperature of only about $400 \mathrm{~K}$. This makes it possible to use the same substrate repeatedly and to ensure reproducible preparation of the sample as well as reproducible desorption signals.

As an example, Fig. 1a displays a kinetic energy distribution obtained by desorbing dimers with laser light of $\lambda=532 \mathrm{~nm}$. The Na coverage was $2.2 \cdot 10^{15}$ atoms $/ \mathrm{cm}^{2}$. This corresponds to an average particle size of $\mathrm{R}_{\mathrm{ave}}=$ $12.6 \mathrm{~nm}$. The fluence of the $\mathrm{Nd}: \mathrm{YAG}$ laser was set to a value of $\Phi=10 \mathrm{~mJ} / \mathrm{cm}^{2}$. As can be seen from the measured distribution, dimers with very low kinetic energies substantially contribute to the signal. Furthermore, the desorption rate falls off rapidly as a function of kinetic energy, a dependence that can be fit to an exponential. It is represented by the dashed line. The corresponding temperature is also given in Fig. 1a. The total amount of dimers desorbed per laser pulse at $\lambda=532 \mathrm{~nm}$ rises sharply with increasing fluence and follows an exponential, see Fig. 1b.

Figure 2 displays a series of measurements with a wavelength of $\lambda=355 \mathrm{~nm}$ and a total coverage of $7.2 \cdot 10^{14}$ atom $/ \mathrm{cm}^{2}\left(\mathrm{R}_{\text {ave }}=7 \mathrm{~nm}\right)$. The laser fluence was increased first from $\Phi=3 \mathrm{~mJ} / \mathrm{cm}^{2}$ (Fig. 2a) to a value of $\Phi=5 \mathrm{~mJ} / \mathrm{cm}^{2}$ (Fig. 2b). In both cases the kinetic energy distribution shows a maximum. In contrast, the distribution changes drastically at larger fluences of, for example, $\Phi=9 \mathrm{~mJ} / \mathrm{cm}^{2}$ (Fig. 2c). It does not exhibit a maximum anymore but falls off monotonously. In fact, an exponential dependence similar to the one observed at a wavelength of $\lambda=532 \mathrm{~nm}$ is obtained.
In further measurements the total coverage and the laser fluence at $\lambda=355 \mathrm{~nm}$ were varied systematically. At low laser fluences a maximum is observed for all kinetic energy distributions of the desorbed dimers. The data are compiled in Fig. 3. It displays the positions of the maxima of the distributions for five values of the laser fluence and four different coverages. The most essential result is that the positions of these maxima at $\mathrm{E}_{\text {kin }}=0.06(1) \mathrm{eV}$ are identical within the experimental error and neither depend on particle size nor on laser fluence.

Figure 4 shows the integral desorption rate, obtained with $\lambda=355 \mathrm{~nm}$ as a function of laser fluence for the fixed coverage of $3.6 \cdot 10^{15}$ atoms $/ \mathrm{cm}^{2}\left(R_{\text {ave }}=15 \mathrm{~nm}\right)$. At low values, the integral desorption rate is linearly dependent on the laser fluence. Above approximately $\Phi=7 \mathrm{~mJ} / \mathrm{cm}^{2}$, however, it grows exponentially. At the same time, the shape of the kinetic energy distribution changes from a curve exhibiting a maximum to an exponential decrease, see Fig. $2 b$ and $2 c$.

We first conclude from the data, that desorption of sodium dimers $\left(\mathrm{Na}_{2}\right)$ is readily detectable for laser wavelengths of $\lambda=355 \mathrm{~nm}$ as well as $532 \mathrm{~nm}$. For laser light of $\lambda=532 \mathrm{~nm}$ the measured kinetic energy distributions are exponential and characteristic of thermal bond breaking. This is supported by the exponential dependence of the integral desorption rate on the laser fluence (Fig. 1b). In contrast, desorption of $\mathrm{Na}_{2}$ with $\lambda=355$ and low fluences $\left(\Phi \leqslant(7 \pm 1) \mathrm{mJ} / \mathrm{cm}^{2}\right)$ obviously occurs as a non-thermal reaction. This is supported by the shape of the kinetic energy distributions of the detected dimers, the linear fluence dependence as well as by the constant value of the most likely kinetic energy of $\mathrm{E}_{\mathrm{kin}}=0.06(1) \mathrm{eV}$. This value turns out to be independent of coverage, i.e. particle 


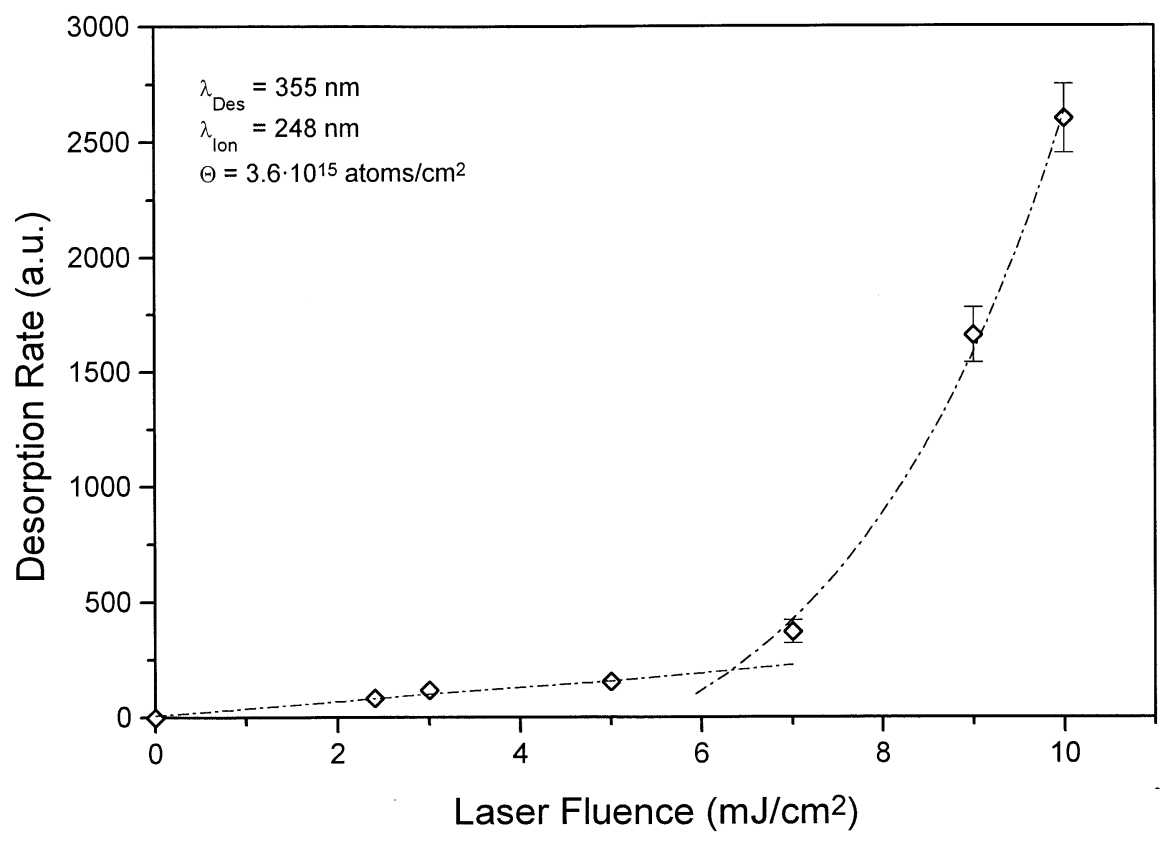

Fig. 4. Integral desorption rate of Na-dimers desorbed with a laser wavelength of $\lambda=355 \mathrm{~nm}$ as a function of laser fluence. The coverage was $3.6 \cdot 10^{15}$ atoms $/ \mathrm{cm}^{2}\left(R_{\text {ave }}=12 \mathrm{~nm}\right)$. The fluence was varied between 1 and $10 \mathrm{~mJ} / \mathrm{cm}^{2}$

size, and, as mentioned above, of the light intensity. Furthermore, the Na particles only experience a moderate temperature rise under the conditions quoted above [13]. Also, the transition from non-thermal to thermal desorption as a function of increasing laser fluence can be identified. It is reflected in the appearance of exponential kinetic energy distributions that gradually replace the curves exhibiting a maximum (Fig. 2) and in the simultaneous transition from a linear to an exponential dependence of the integral desorption rate (Fig. 4). This agrees very well with the results of earlier work [5], which showed thermal desorption of the sodium dimers for higher laser fluences.

The results reported in the present paper indicate that the dimers are directly desorbed from the surface and not formed by gas phase collisions of the desorbing atoms. The following two arguments lead to that conclusion. In order to form the dimers by gas phase collisions in front of the surface three body collisions would be required. They occur, however, with sufficient probability only at large sodium vapour pressure that can not be produced by the low laser fluences used in this experiment. In addition, three body collisions would lead to strong thermalization of the kinetic energy distributions of the sodium dimers and atoms [22], in strong contrast to the observed energy distributions of the Na-dimers reported in the present paper and of Na-atoms published earlier [19].

The large ratio of the desorption rates of dimers and atoms (factor 3-4) indicates that the dimers obviously constitute highly stable subunits of the metal surface that can be released in a photochemical reaction without breaking into individual atoms. This seems to imply that the dimers are oriented on the surface in such a way that the axis connecting the two Na-atoms is not parallel to the substrate surface but rather points into the direction of the surface normal. The repulsive electronic excitation preceeding desorption must than be localized in the region between the dimer and the remaining substrate atoms.

In future experiments the non-thermal reaction as well as the interplay between thermal and non-thermal bondbreaking will be studied in more detail. For example, the angular distribution of the desorbed atoms and dimers should give further information on the desorption mechanism. A very interesting question is also, whether or not desorption of aggregates $X_{n}$ with $n>2$ can be stimulated. In order to address this problem, use of longer ionization wavelengths is essential to prevent photofragmentation of the larger aggregates [23].

Acknowledgements. Financial support of the Fond der Chemischen Industrie (F.T.) and BMBF 13N 61597 (M.S.) is gratefully acknowledged.

\section{References}

1. A. Benninghoven, F.G. Ruedenauer, H.W. Werner:New York, Wiley 1987

2. P.D. Brewer, M. Späth, M. Stuke: Mat. Res. Soc. Symp. Proc. 334, 245 (1994)

3. M. Wolf, T. Hertel, G. Ertl: Nucl. Instru. Meth. Phys. Res. B 101, 56 (1995)

4. R.W. Dreyfus: Appl. Phys. A 55, 335 (1992)

5. T. Götz, M. Bergt, W. Hoheisel, F. Träger, M. Stuke: Appl. Surf. Sci. 96-98 (1996), 280

6. J.A. Prybyla, T.F. Heinz, J.A. Misewich, M.M. Loy, J.H. Glownia: Phys. Rev. Lett. 64, 1537 (1990)

7. D.G. Busch, S. Gao, R.A. Pelak, M.F. Booth, W. Ho: Phys. Rev. Lett. 75, 673 (1995)

8. F. Budde, T.F. Heinz, M.M. Loy, J.A. Miswich, F. de Rougemont, H. Zacharias: Phys. Rev. Lett. 66, 3024 (1991)

9. P. Voumard, R. Zenobi: J. Chem. Phys. 103, 6795 (1995) 
10. X.Y. Zhu, J.M. White: Phys. Rev. Lett. 68, 3359 (1992)

11. L. Phillippe, G. Dujardin, M.J. Besnard Ramage, L. Hellner, T. Hirayama, G. Comtet, M. Rose: Nucl. Instru. Meth. Phys. Res B 101, 200 (1995)

12. A. Drescher, J. Kitching, J.E. Crawford, J.K.P. Lee, G. Thekkadath: Z. Physik D 19, 203 (1991)

13. W. Hoheisel, M. Vollmer, F. Träger: Phys. Rev. B 48, 17463 (1993)

14. W. Hoheisel, K. Jungmann, M. Vollmer, R. Weidenauer, F. Träger: Phys. Rev. Lett. 60, 1649 (1988)

15. A.M. Bonch-Bruevich, T.A. Vartanyan, Yu.N. Maksimov, S.G. Przhibel'skii, V.V. Khromov: Surf. Sci. 307-309, 350 (1994)
16. H.S. Kim, H. Helvajian: J. Chem. Phys. 95, 6623 (1991)

17. M.J. Shea, R.N. Compton: Phys. Rev. B 47, 9967 (1993)

18. K. Ishikawa, J. Kawasaki, Y. Nakai, M. Itoh: Surf. Sci. 349, L152 (1996)

19. T. Götz, M. Bergt, W. Hoheisel, F. Träger, M. Stuke: Appl. Phys. A, 63, 315 (1996)

20. J.A. Venables: Surf. Sci. 299/300, 798 (1994)

21. T.D. Bennett, D.J. Krainovich, C.P. Grigoropoulos: Phys. Rev. Lett 76, 1659 (1995)

22. D. Sibold, H.M. Urbassek: Phys. Rev. A 43, 6722 (1991)

23. K.J. Peterson, P.D. Dao, R.W. Farley, A.W. Castleman: J. Chem. Phys. 80, 1780 (1984) 